\title{
Multidimensional imaging for skin tissue surface characterization
}

\author{
Jiuai Sun, Melvyn Smith \\ Machine Vision Lab, University of the West of England, Bristol, UK, BS16 1QY
}

\begin{abstract}
Human skin, the outer and largest organ covering our body, can be described in terms of both its 3D spatial topography and its 2D spectral reflectance. Such a characterization normally requires the application of separate procedures using different kinds of equipment, where spectral reflectance can only be obtained from a small patch of the skin surface. This paper investigates the integration of multiple imaging modalities to simultaneously capture both spectral and spatial information from the skin surface over a wide area. By extending the imaging spectrum from the visible to the near-infrared (NIR), we improve general recovery, obtain a more detailed skin profile, and are able to identify the distribution of various principal chromophores within the deeper dermal layers. Experiments show that new dimensions of skin characterization can be generated through the recovered geometrical and spectral information, so that an enhanced visibility of important skin physiological phenomena can be achieved.
\end{abstract}

Keywords - skin, spectral, shape, photometric stereo, chromophore, wide-field

\section{Introduction}

Covering our entire body, our skin allows us to interact with the external environment, while at the same time protecting us from environmental damage - for example through mechanical friction, noxious chemicals, radiation exposure or temperature extremes. Our skin appearance also serves to reflect internal changes, such as mood, aging and various pathologies relating to infection and metastasis. Although these mechanisms are is still not fully understood, knowledge concerning dermal molecular and skin anatomical structures have been understood and found useful in characterizing certain functional and cosmetic skin attributes in various applications. For example, some cosmetic products aim to moisturise and so smooth the skin microstructure to achieve a healthy and attractive impression. Computer graphics and vision experts also use knowledge concerning the skin structure to better model its reflective properties in order to achieve more realistic rendering of human faces or in identifying individuals [1].

The skin surface at a micro-scale is not simply flat but able to be characterized by a particular relief format, representing the evolving keratinisation progress of the epidermis 
and general 3D organization of dermis and subcutaneous tissue [2]. The periodical cellular updating and frequent mechanical interaction with the external environment give skin a net-like topographic profile composed of regular triangles, polygons and line patterns, which may later evolve progressively into large scale wrinkles. The roughness and palpation parameters obtained from this pattern of micro-relief has been used for evaluating the effectiveness of cosmetic products and in assessing the status of tumours beneath the skin surface [3]. The skin subsurface is itself rich in various pigments whose interaction with incident photons gives skin its colourful impression. Among these are melanin and haemoglobin - the two main chromophores embedded within the epidermal and dermal layers respectively. These have their own selective absorption and scatter characteristics at different regions of the light spectrum. The optical characteristics, spatial density and distribution of these chromophores jointly determine the exact skin reflectance/colour. The reflectance/colour is most accessible and is frequently used for assessing relevant skin physiological phenomena, such as an erythema caused by blood perfusion of burnt/grafted tissue, or a variegated pigment lesion produced by a deeper invasion of malignant melanin [4]. To objectively characterize the skin profile or its reflectance information, several approaches have already been developed.

Skin surface profiles are mainly obtained through mechanical or optical techniques. Mechanical profilometry works by moving and recording a stylus across a silicone polymer replica of the skin surface and then reconstructing the surface profile from the recorded data of the vertical movements of the stylus. Although this has been used in the field for several years, it should be noted that the contact pressure from the weight of the stylus may introduce some deformation as the stylus moves across the soft silicon replica. It is also a time-consuming procedure to move the stylus in very small steps to achieve high resolution measurements [5] and of course there is the need to produce the silicon replica in the first place. In contrast to mechanical techniques, the 'stylus' used in optical various methods take the form of spots, lines or patterns of structured lights. For example, a time multiplexing optical system frequently used in dermatology projects a series of parallel white stripes with a constant phase shift onto the tissue surface from which the 3D topography is calculated based on the observed deflection of the stripes [6]. There is no direct contact pressure on the skin in the case of optical measurement techniques and this is an important consideration that serves to make optical measurement techniques more appealing. Moreover, in addition to the skin profile, an optical approach offers the potentiality to capture skin reflectance, another important dimension in characterizing the skin surface.

The reflectance/colour of skin is normally measured through spectrometers or tristimulus colorimeters. Spectrometers capture the skin spectral response within a number of spectral bands over a relatively small skin area, and the reflectance of the skin is calculated from the collected abundant spectral response data. The spectral bands are normally selected to be able to reflect concentrations of melanin and haemoglobin, according to their absorption and scattering characteristics [7]. The approach can achieve some reproducibility in quantifying skin reflectance by constraining the light spectrum and the working environment, though it is not a format offering a direct visual impression. Tristimulus colorimeters quantify skin colour through specifying the 
illumination and viewing standards which are able to represent all possible perceivable colours in three values, i.e. the tristimulus system is based on the International Commission on Illumination (CIE) standard. This enables measurements obtained using a tristimulus colorimeter to be more traceable and are usefully comparable to that perceived by the human eye. It has also been verified that readings from colorimeters are able to correlate with those from spectrometers. However both spectrometers and tristimulus colorimeters have difficulties in quantifying skin reflectance over a relative large area $\left(\geq 5 \mathrm{~cm}^{2}\right)$, since they need to assume the area inspected is flat and homogeneous [8].

Measuring skin profile and reflectance separately in the above methods may be problematic, especially for a relatively large area, where skin topography is neither flat nor of uniform reflection. This is because the appearance of skin is jointly determined by both the intrinsic variables of the skin surface profile and also its reflectance. A reflection bias can easily be produced when measuring curved body regions if the shape factor has not been taken into account. Such bias could then be transferred into the calculation of the real distribution of optical features for quantifying the physiological status of the skin [9]. In order to remove artifacts caused by surface curvature, an additional 3D imaging modality is often used to recover an object's shape for reliable spectral mapping measurements. For example, the multiplexing optical system mentioned above, along with other structured lighting approaches, have been employed to capture the skin profile for removing intensity artifacts $[6,10,11]$.

To reconstruct the skin micro-relief using structured lighting approaches requires high spatial resolution of any light stripe pattern. Difficulties may be experienced in scanning heavily pigmented lesion such as melasma and melanoma as the projected strips will tend to appear blended with the pigmentation of the skin. In order to solve these problems we have proposed a photometric stereo (PMS) technique to recover the topographic shape of the skin tissue by using multiple images, illuminated from different directions, but viewed from one single location [12]. Because most photons entering the skin and interacting with the chromophores will undergo hundreds of absorptions and scatterings before emitting from the skin, the spatial distribution of the radiation becomes isotropic. Also, the micro-facet structure of the stratum corneum makes the skin somewhat diffusive [13]. Therefore a photometric stereo approach based on a diffusive reflection model is appropriate for extraction of both the surface reflectance and shape, and suffers less from any coloured texture at the tissue surface.

This paper will extend our early work and aims to exploit the synergy of a multimodal approach by combining a multispectral capability with photometric stereo imaging technology for the analysis of skin tissue reflection and geometrical dimensioning within the visible and NIR spectrum. As far as we are aware, only a few systems have tried to fuse these two separate forms of information $[14,15]$. Superior to the existing systems, the combined multispectral imaging and the PMS in our approach, share a single lighting path, therefore the geometrical and spectral reflection measurements are aligned exactly, without involving any extra registration procedure. This means that the shape factor used in calculating spectral mapping can be easily eliminated. Furthermore, we also later 
recombine the recovered spectral and geometrical information together in order to enhance the visibility of more subtle physiological phenomena.

\section{Methodology}

\subsection{Simplified skin optical reflection model}

Skin is commonly recognized as a thin structure with distinctive multiple sub-layers, often simplified into epidermis, dermis and the basal collagen/fat layer [1, 13]. When a beam of white light is projected onto this thin structure, most of the light will enter the skin and interact with unique biological chromophores embedded within the layered anatomical structures. More specifically, the light firstly passing through the epidermis is absorbed by melanin, which demonstrates a higher absorptive coefficient at shorter wavelengths. The total amount of this absorption is determined by the range of the illumination spectrum, together with the concentration and distribution of melanin. Then the light further penetrating into the dermis interacts with the hemoglobin pigments and collagen fibers in the form of absorption and scatter. Finally, the basal collagen/fat layer under the dermis acts as a highly diffusive surface, approximately independent of wavelength.

The above simplification of the skin optical transportation mechanism allows the development of a three layer optical model, i.e. two uniform media layers over an ideal diffusive surface [16]. The first and second layers are the epidermis and dermis, and the lowest surface is equivalent to a perfect diffusive fat layer, containing no chromophores. The direct reflection on the epidermis surface and the interface between the epidermis and dermis are negligible. Although light scatter does exist, especially within the dermal layer, this optical model assumes absorption as the single most significant manner of light transportation within the two layers. This simplified theory has been successfully applied to explain and detect important physiological phenomena.

The simplified skin reflection $R_{\text {skin }}$ can be represented as:

$R_{\text {skin }}^{\lambda}=T_{\text {epid }}^{\lambda{ }^{2}} T_{\text {derm }}^{\lambda}{ }^{2} R_{\text {diff }}^{\lambda}$

where $T_{\text {epid }}^{\lambda}$ and $T_{\text {derm }}^{\lambda}$ are the transmittance coefficients of the simulated epidermis and dermis layers; the square operator means the lights pass through the layers two times; and $R_{\text {diff }}^{\lambda}$ is the diffusive reflectance coefficient of the deepest fat layer.

The transmittance through transparent layer satisfies the Lambert-Beer law, i.e. 
$T_{\text {epid }}^{\lambda}=e^{-\mu_{m}^{\lambda} l_{\text {epid }}{ }^{*} c_{\mathrm{m}}}$

$T_{\text {derm }}^{\lambda}=e^{-\mu_{h}^{\lambda} * l_{\mathrm{derm}}{ }^{*} c_{\mathrm{h}}}$

where $\mu_{m}^{\lambda}$ and $\mu_{h}^{\lambda}$ are the wavelength-dependent absorptive coefficients of melanin and hemoglobin; $l_{\text {epid }}$ and $l_{\text {derm }}$ are the length of light paths within epidermis and dermis; and $c_{m}$ and $c_{h}$ are the concentrations of melanin and hemoglobin chromophores respectively.

Therefore, the general reflectance of the skin surface at the wavelength $\lambda$ is:

$R_{\text {skin }}^{\lambda}=\left(T_{\text {epid }}^{\lambda}\right)^{2}\left(T_{\text {derm }}^{\lambda}\right)^{2} R_{\text {diff }}^{\lambda}=e^{-2\left(\mu_{m}^{\lambda} l_{\text {epid }} c_{m}+\mu_{h}^{\lambda} l_{\text {derm }} c_{h}\right)} R_{\text {diff }}^{\lambda}$

Incorporating knowledge concerning the penetration depth of the incident light and the unique absorption patterns of melanin and hemoglobin, the above expression can be further simplified at a specific range of wavelengths. For example, the approximate depths of light penetration on a fair Caucasian skin type at typical wavelengths of $450 \mathrm{~nm}$ (blue), 500nm (green) and $600 \mathrm{~nm}$ (red) are $150 \mu \mathrm{m}, 230 \mu \mathrm{m}$ and $550 \mu \mathrm{m}$ respectively. However, the thickness of the epidermis and dermis are around $100 \mu \mathrm{m}$ and $200 \mu \mathrm{m}$. Therefore the blue light is just able to penetrate through the epidermal layer, while the green light nearly reaches the diffusive reflectance layer. Note that the absorbance of hemoglobin at a longer wavelength becomes weak, so that only the melanin effect need be considered in that case.

\subsection{Multiple spectral photometric stereo}

A Lambertian model can be used to approximate the reflection properties of human skin as the skin exhibits a predominately diffusive characteristic after the specular reflection is removed. Therefore the intensity of light reflected from the skin surface can be expressed as:

$I^{\lambda}=\xi^{\lambda} R_{s k i n}^{\lambda}(\boldsymbol{l} \cdot \boldsymbol{n})=\xi^{\lambda} R_{\text {skin }}^{\lambda} \cos (\theta)$

Where $\xi^{\lambda}$ is a composite factor determined by the intensity and spectral of the illumination together with the sensitivity of the optical sensor, which can be calibrated for by using a standard white flat reflectance object [17]; $R_{\text {skin }}^{\lambda}$ is the skin reflectance, sometimes called as absolute spectral reflectance, which can be treated as a combination of transmittance and diffusive reflection [18]; $\boldsymbol{l}$ and $\boldsymbol{n}$ are the incident light direction and skin surface normal; and $\theta$ is the angle between surface normal and light direction. 
In order to recover skin surface orientation and reflectance, one potential way is to take more than one image using the same imaging system from the same observation point, but illuminated by a single light source from a different direction $\boldsymbol{l}_{j}(j=1,2, \ldots, \mathrm{N}, \mathrm{N} \geq 3)$. Therefore a grouping of the equation (5) can be found and rewritten as:

$\boldsymbol{I}^{\lambda}=\xi^{\lambda} R_{\text {skin }}^{\lambda}(\boldsymbol{L} \cdot \boldsymbol{n})$

where $\mathbf{L}$ is the illumination matrix, formed by arranging the individual light directions as $\boldsymbol{L}=\left(\boldsymbol{l}_{1}, \boldsymbol{l}_{2}, \cdots, \boldsymbol{l}_{N}\right)^{T}$. Similarly a vector of image intensity can be compiled as $\boldsymbol{I}_{j}=\left(I_{1}, I_{2}, \cdots, I_{N}\right)^{T}$. The unknown reflectance and surface normal can now be uniquely calculated through a least-squares method, providing the calibrated illumination matrix $\mathbf{L}$ is available. This is known as a traditional photometric stereo approach. Due to accessibility of colour photography, an improved solution has been achieved through averaging the traditional PMS results across three channels [12].

Theoretically the surface normal as calculated using the three separate colour channels should be similar and its average should be closer to a true value. Similarly the calculated reflectance should be consistent and independent from the illumination spectrum [18]. However, the major light transportation within the skin is all wavelength dependent due to the variations of optical transmission characteristics and anatomical structure of the skin subsurface. Therefore the characterization of the skin surface should be treated differently at each channel if a colour camera is used.

\subsection{Chromophore separation with additional spectral response}

As light reflected from the skin is dependent on the illumination spectrum and optical characteristics of the chromophores with respect to the spectrum, each image taken under each specific lighting condition will be different from each other, though the biological and anatomical features underneath skin remain the same. Given the availability of the spectral response at redundant channels, some biological features, such as the concentration of chromophores, may be extracted.

As the light attenuation at longer a wavelength is dominated by the absorption of melanin, while the absorption caused by the hemoglobin is negligible, expressions (4) and (5) can be further simplified into:

$$
\begin{aligned}
& I^{\text {inf }}=\xi^{i n f} e^{-2 \mu_{m}^{i n f} l_{\text {epid }} c_{m}} R_{\text {diff }}^{\text {inf }} \cos (\theta) \\
& I^{r \varepsilon d}=\xi^{r \varepsilon d} e^{-2 \mu_{m}^{r e d} l_{\text {epid }} c_{m}} R_{d i f f}^{r e d} \cos (\theta)
\end{aligned}
$$




$$
\begin{aligned}
& I^{\text {green }}=\xi^{\text {green }} e^{-2\left(\mu_{m}^{\text {green }} l_{\text {epid }} c_{m}+\mu_{h}^{\text {green }} l_{\text {derm }} c_{h}\right)} R_{\text {diff }}^{\text {gresn }} \cos (\theta) \\
& I^{\text {blue }}=\xi^{\text {blue }} e^{-2\left(\mu_{m}^{\text {blue }} l_{\text {epid }} c_{m}+\mu_{h}^{\text {blue }} l_{\text {derm }} c_{h}\right)} R_{\text {diff }}^{\text {blue }} \cos (\theta)
\end{aligned}
$$

The concentration of melanin $c_{m}$ is readily deduced by dividing equation (7) by (8), providing the absorption coefficient and depth of penetration of melanin are prior known. It is also worth noting that the factor of surface shape is cancelled out automatically due to this division operation. Therefore the melanin component is able to be calculated from the response of two long wavelengths (i.e. red and NIR). This conclusion agrees with early work [21].

The concentration of hemoglobin $c_{h}$ can be recovered by substituting the calculated melanin concentration into equation (9). The response at a blue wavelength has not been used because the penetration capability of blue light in the dermal layer is uncertain.

\subsection{Viewing blood vessels through the NIR spectrum}

A new combination of spectral reflections at different wavelengths may be used to provide a unique impression of human skin, able to usefully reveal the subtle intensity and distribution of interesting skin chromophore components. In previous related work, NIR images have been fused with visible images by professional photographers and remote surveyors in order to identify particular plants and vegetable regions $[19,20]$. The depth dependent absorptions of chromophores has also been obtained from skin reflectance captured by a multispectral transillumination imaging device [22]. Similarly, here we use both recovered spectral and shape information, integrated to enhance interesting physiological conditions of the skin tissue surface.

The NIR spectrum can penetrate deeper into skin than the visible spectrum because the absorption and scatter effect within the optical window is weaker. Also the relative high density of the blood component within the blood vessels absorbs a greater degree of the incident light. Therefore a high contrast image of the blood vessels can be obtained by employing NIR illuminations. The $850 \mathrm{~nm}$ NIR light used in our new imaging approach approximates to those optimal wavelengths necessary for viewing the vascular structure [23]. In contrast to others who have used a mercury arc lamp for general spectral illumination and structured lighting for obtaining the 3D topography of the object surface [11], here four pairs of visible and NIR LEDs are sequentially triggered to project light onto the skin surface in our new imaging approach (Fig 1(a)). An enhanced colour blood vessel image is obtained by fusing the base layer of the NIR image with that of a visible image, calculated from a bilateral filter [24]. After suitable enhancement, even those fine blood vessels with small size below the pigmented skin area can be clearly visually identified. 


\section{Experiments}

\subsection{Instrumental setup}

A mult-spectral camera (JAI's AD-080GE) shown in Fig. 1(a) has been used as the optical sensor for building the new imaging system. Fig. 1(b) demonstrates how the new system works: a prism built in to the camera splits the incident light into both the visible and NIR spectrum; two separated light sources are projected onto two CCD sensors with same resolution but different spectral response; reflection and geometrical properties are recovered from the PMS approach; finally, meaningful physiological phenomena are revealed through different visualization and enhancement pipelines.

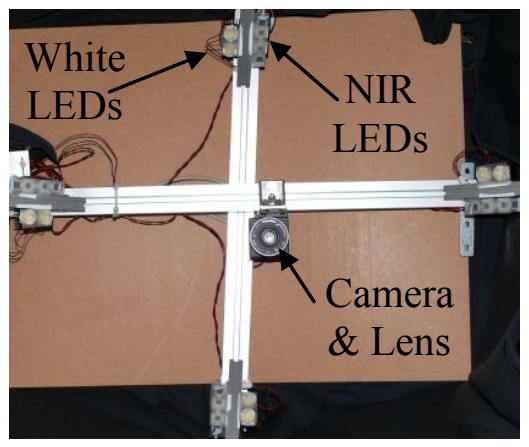

(a) The system consists of four pairs of white and NIR LEDs, one 2CCD camera and one Schneider lens

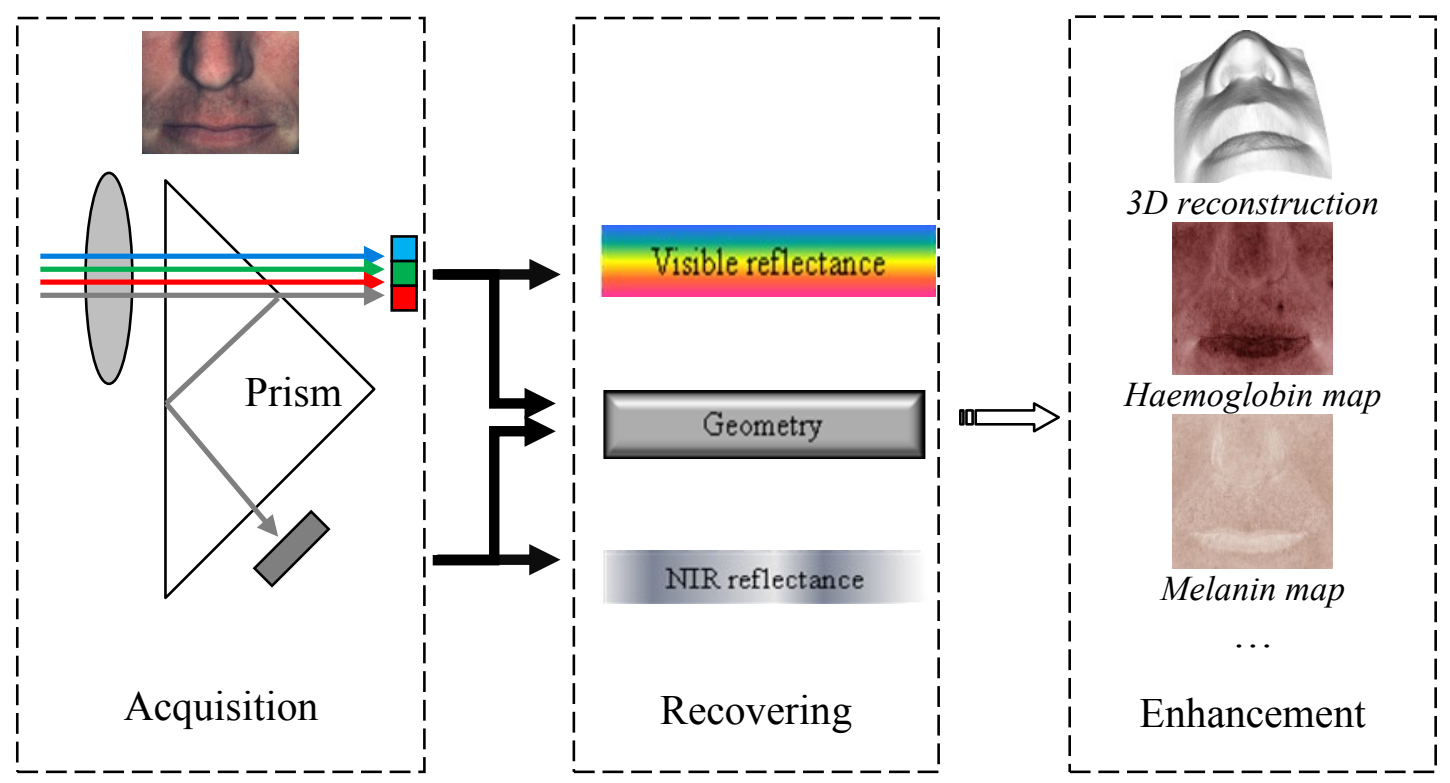

(b) Working flow chart

Figure 1 Setup (a) and schematics (b) of the new imaging modalities 
For implementing multiple spectral PMS, four pairs of white and NIR LEDs are selected as the lighting sources. A high-resolution Schneider lens is used to collect images using both visible and NIR spectrum lights. The lighting directions relative to optical center are estimated by using a specular sphere with known size [25]. In order to eliminate the composite spectral effect of the illumination and optical sensor, an Edmund white balance target having 99\% uniform reflectance from ultraviolet to NIR spectrum is used to sample the light field. The combination effect of lighting and optical sensor can be compensated for when the objects to be recovered are approximately located around the calibrated space [17].

\subsection{Reflectance recovery and 3D reconstruction}

Previous work has taken the average value of the surface normal recovered across three colour channels as the final solution and then used it to calculate the reflectance at every channel. This experiment investigated the individual channels separately and finds their contribution to the final result. Fig. 2(a) is one image of a volunteer's wrist taken using the new system and Fig. 2(b) is a magnified view of the small skin region outlined by the black solid lines in Fig. 2(a). Four pairs of colour and NIR images are taken for the recovery of the reflectance and surface normal data from the skin surface using the PMS technique.

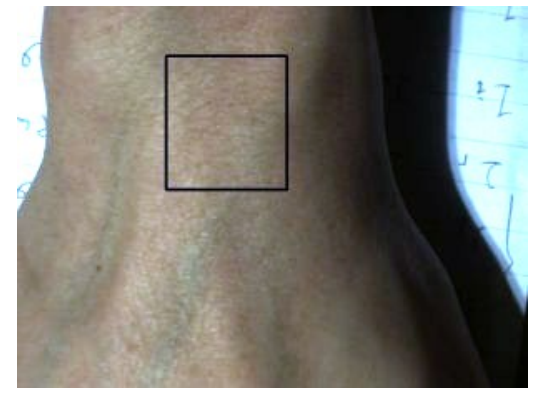

(a)

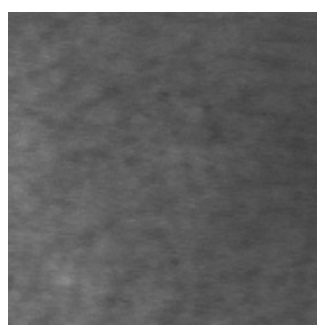

(c)

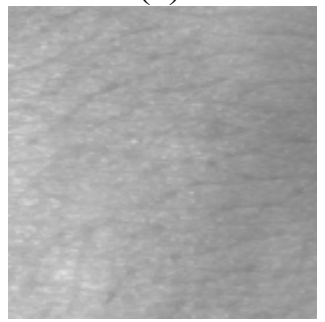

(g)

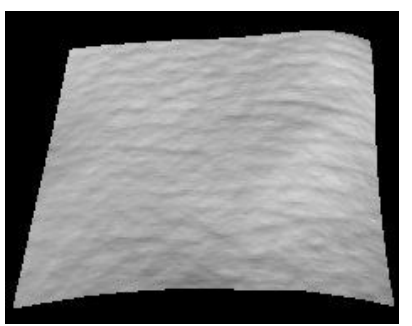

(d)

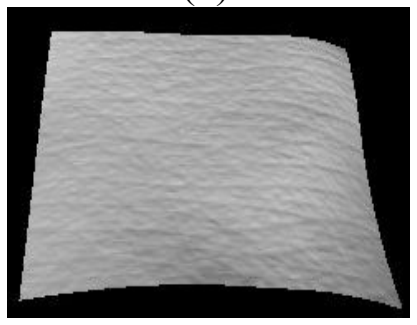

(h)

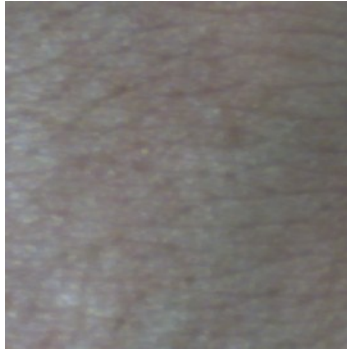

(b)

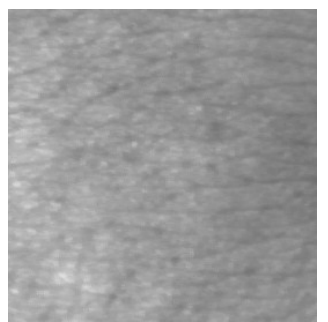

(e)

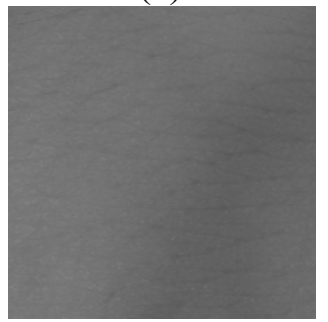

(i)

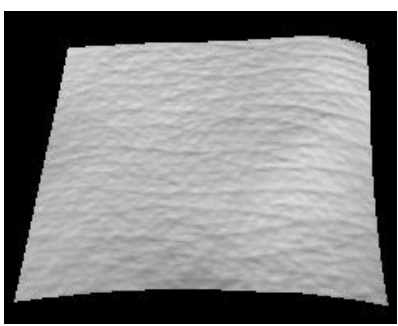

(f)

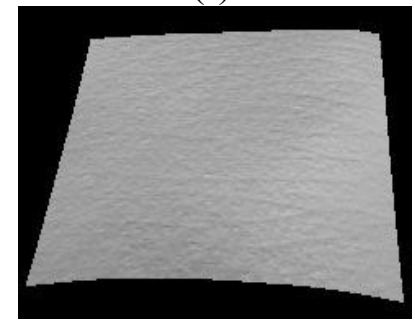

(j) 
Figure 2 A volunteer's wrist (a) with small skin region (b) outlined, its reflectance (c, e, $g, i)$ and $3 D$ surfaces $(d, f, h, j)$ recovered at blue, green, red and NIR channels

The recovered reflectance in Fig. 2(c, e, g, i) demonstrates a noticeable variation across the visible and NIR spectrum. The reflectance maps recovered in the blue and NIR channels look darker than that in green and red channels. This could be caused by the strong absorption of melanin at the shorter wavelength within the epidermis and light scattering effect at a longer wavelength within the dermis. In addition, the NIR reflectance exhibits a more homogenous distribution, which may reflect the uniform diffusion at the collagen/fat layer. The 3D reconstruction results in Fig. 2(d, f, h, j) show that the green and red channels offer good recovery of finer skin line net structures, while the NIR channel can only provide the general smooth shape of the overall skin surface. Therefore, we can see that selection of the green and red spectrum becomes significant for imaging conditions such as wrinkle and psoriasis assessment, where the fine skin structure is of particular interest.

\subsection{Chromophore mappings}

Melanin and hemoglobin embedded within skin surface are the two main chromophores reflecting important skin physiological phenomena, such as skin tanning, expression, alcohol abuse, physical abuse and skin cancers. An objective extraction of the two pigmentation components from photographs across a large area is always desired, as compared to conventional spectrometer approaches working only over a limited and small skin area.

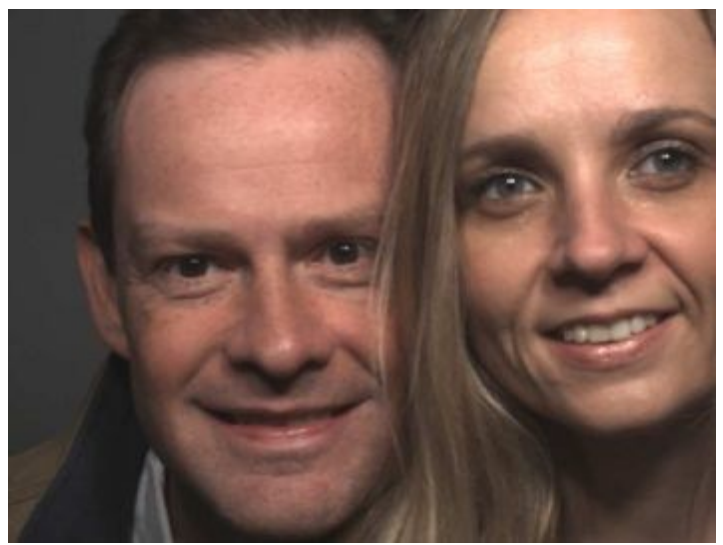

(a)

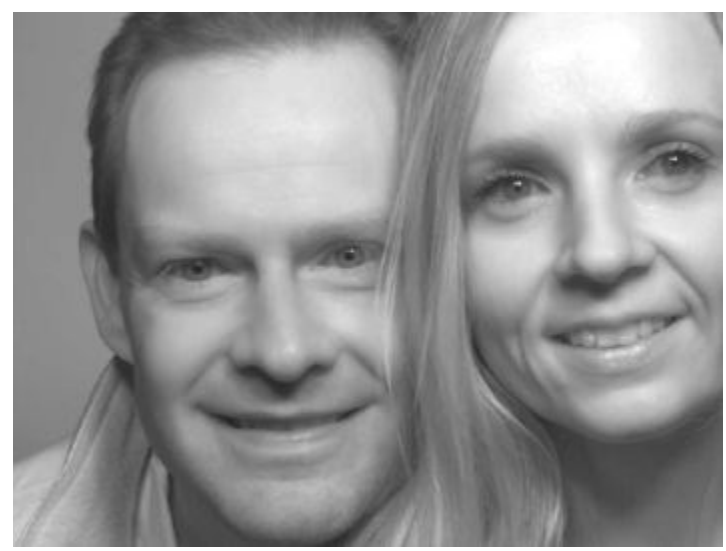

(b) 


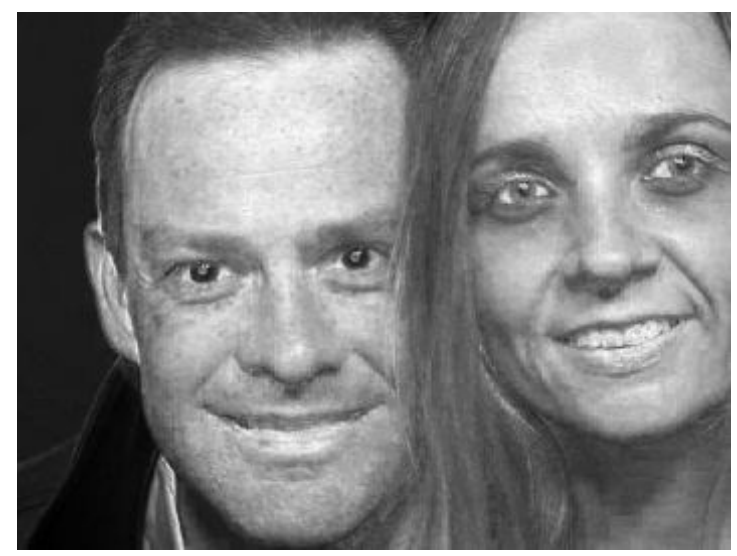

(c)

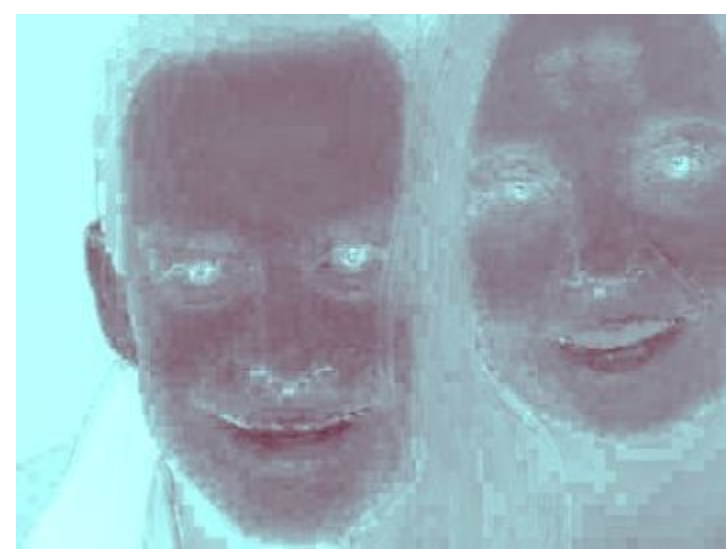

(d)

Figure 3: A photo (courtesy of Prof. Sabine Süsstrunk, EPFL) in the visible (a) and its counterpart NIR spectrum (b), separated melanin (c) and hemoglobin mappings (d)

Figure 3 shows the implementation of the approach described in section 2.3 on photographs captured with the same optical sensor by a different research group [24]. The freckles distributed around the man's forehead and the region under his eyes can be seen more clearly by looking at the separation result in Fig. 3 (c). Similarly, the concentration of hemoglobin can be found to be denser around lips and cheeks from Fig. 3(d). This shows the fusion of the NIR and visible images captured from using a multiple spectral imaging modality can be used to quantitatively isolate aspects of the skin condition (melanin and hemoglobin mappings) that are underneath the skin tissue surface and so normally subtle and without any complicated human intervention.

\subsection{Enhancement and visualization of blood vessels}

Blood vessels within the subcutaneous layer are only poorly visible under white light as most of the incident light is normally attenuated by the absorption of chromophores and dermal scattering before reaching the subcutaneous tissues, including blood vessels. However, the employment of NIR light gives our new imaging modalitie an additional sensitivity, able to reveal subcutaneous anatomic structures deeper within the skin.

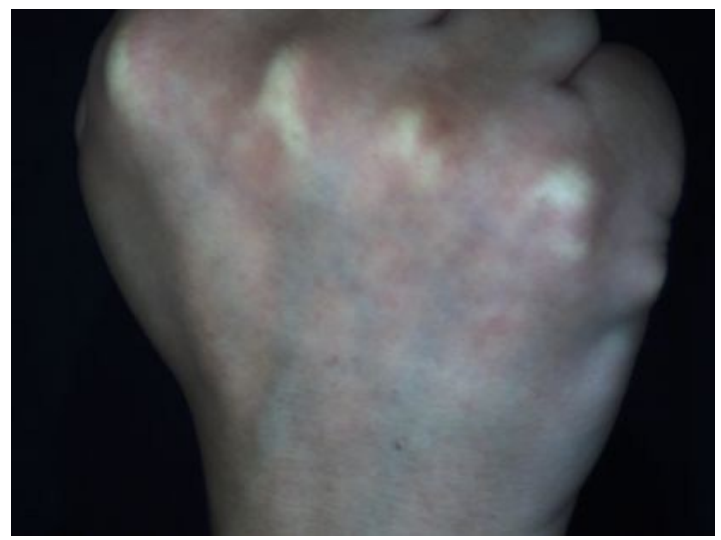

(a)

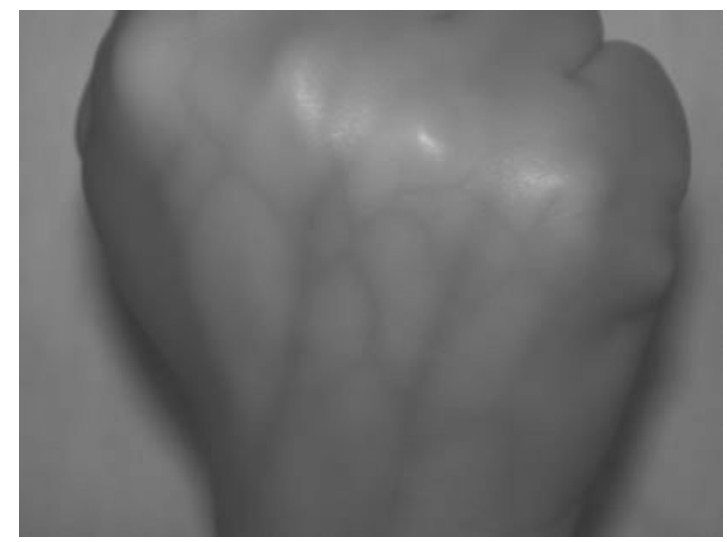

(b) 


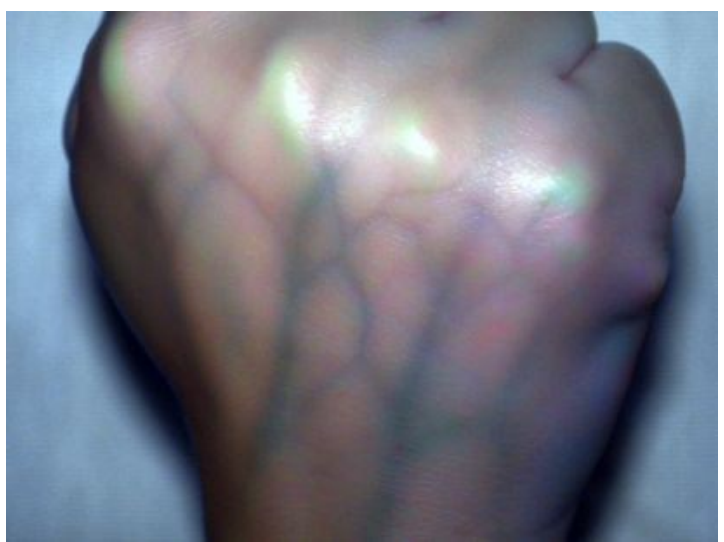

(c)

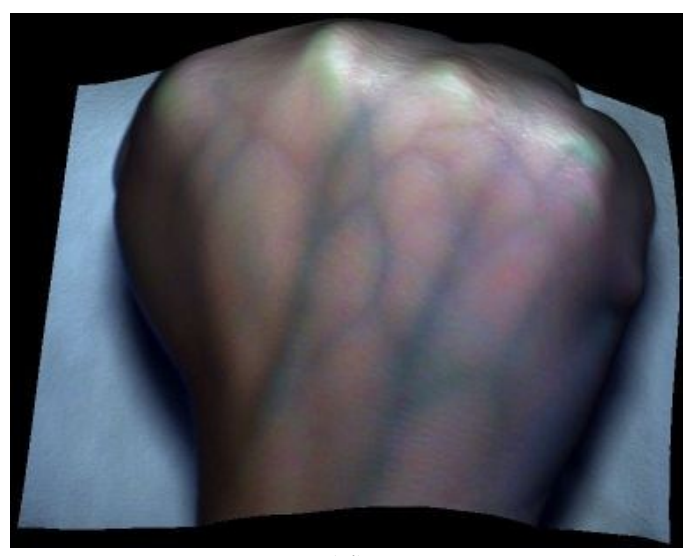

(d)

Figure 4 A fist under visible (a) light and its counterpart NIR spectrum (b), enhanced blood vessel detail (c) and its overlay on the 3D reconstruction result from the PMS (d)

Fig. 4(a) and (b) are images of a volunteer's fist acquired using the new imaging system within the visible and NIR spectrum. It can be seen that the smaller fine blood vessels are hidden and almost invisible under the white lighting condition, while the tree-like structure of the vascular structure is clearly revealed in the colour enhancement format of Fig. 4(c). Finally, the enhanced vessel structure is overlaid onto the 3D surface reconstructed from the PMS approach in Fig. 4(d) to give an enhanced augmented view.

\section{Conclusions}

Due to the complexity of the various light interaction mechanisms, partial translucency and complex sub-surface anatomical microstructures, human skin tissue is significantly more difficult to characterize than most artificial object materials, whose surfaces normally consists of an established composition with well-known optical properties. This paper describes an approach that exploits the synergy of a multimodal approach, combining multispectral and photometric stereo imaging techniques to simultaneously capture both a spectral and geometrical description of the skin. The extracted geometric and spectral information is later synthetically recombined to usefully enhance the visibility of particular physiological conditions associated with human skin. Initial experimental results demonstrate that these new imaging modalities are able to reconstruct skin tissue topography registered with various enhanced spectral information. This demonstrates the potentiality of the new approach for applications in the biomedical, forensic and computer graphics fields, such as documenting and assessing the tissue surface, assisting in tissue feature catheterization and guiding endoscopic interventions requiring enhanced or augmented visibility of fine surface and subsurface features.

Although the approach has been verified as effective in recovering the geometry and spectral reflection of the skin tissue surface over a relative large area, and enhancing the visibility of chromophore maps by fusing the recovered information together, further work is still required to quantitatively evaluate its sensitivity to melanin, hemoglobin and other important chromophores. 


\section{Reference}

[1] T. Igarashi, K. Nishino, S.K. Nayar, The appearance of human skin, Technical Report, Dept. of Computer Science, Columbia University, CUCS-024-05, 2005

[2] J. L. Leveque, EEMCO guidance for the assessment of skin topography, J. Eur. Acad. Dermatol. Venereol, 12(1999), pp. 103-114

[3] M. Egawa, M. H. Oguri, M. Takahashi, M. Miyakawa, The evaluation of skin friction using a frictional feel analyzer, Skin Res Technol, 8 (2002), pp. 41-51

[4] Z. Liu, J. Sun, L. Smith, M. Smith, R. Warr, Distribution quantification on dermoscopy images for computer-assisted diagnosis of cutaneous melanomas, Med. Biol. Eng. Comput, 50(5) (2012), pp. 503-513

[5] K. Articus, G. Khazaka, K.P. Wilhelm, The Skin Visiometer - A photometric device for the measurement of skin roughness; in K. P. Wilhelm, P. Elsner, E. Berardesca, H. I. Maibach (eds): Bioengineering of the Skin: Skin Surface Imaging and Analysis, Boca Raton, CRC Press, (1997), pp. 59-71

[6] S. Jaspers, Rapid in vivo measurement of the topography of human skin by active image triangulation using a digital micro-mirror device, Skin Res. Technol. 5(3) (1999), pp. 195-207

[7] G.N. Stamatas, B.Z. Zmudzka, N. Kolias, J.Z. Beer, Non-invasive measurements of skin pigmentation in situ, Pigment Cell Res., 17(6) (2004), pp. 618-26

[8] H. Takiwaki, L. Overgaard, J. Serup, Comparison of narrowband reflectance spectrophotometric and tristimulus colorimetric measurement of skin color - 23 anatomical sites evaluated by the DermaSpectrometer and the Chroma Meter CR-200, Skin. Pharmacol., 7 (1994), pp. 217-225

[9] J.M. Kainerstorfer, et. al, Direct curvature correction for noncontact imaging modalities applied to multispectral imaging. J Biomed Opt, 15(4)(2010) 046013

[10] M. Westhauser, et. al, Optimizing color reproduction of a topometric measurement system for medical applications, Med. Eng. Phys., 30 (8) (2008), pp. $1065-1070$

[11] V.C. Paquit, K.W. Tobin, J.R. Price, F. Meriaudeau, 3D and multispectral imaging for subcutaneous vein detection, Opt Express, 17 (2009, pp. 11360-11365

[12] J. Sun, M. Smith, L. Smith, L. Coutts, R. Dabis, C. Harland, J. Bamber, Reflectance of human skin using color photometric stereo: with particular application to pigmented lesion analysis, Skin Res. and Tech., 14(2) (2008), pp. 173-179

[13] A. Krishnaswamy, G.V.G. Baranoski, A study on skin optics. Technical Report CS-2004-01, School of Computer Science, University of Waterloo, Canada, 2004.

[14] G.N. Stamatas, et. al, In vivo monitoring of cutaneous edema using spectral imaging in the visible and near infrared. J. Invest Dermatol., 126(8) (2006), 1753-60

[15] J.M. Kainerstorfer, P. Smith, A.H. Gandjbakhche, Non-contact wide-field multispectral imaging for tissue characterization, IEEE Journal of Selected Topics in Quantum Electronics, 18(4) (2012), pp. 1343-1354

[16] B. Dawson, J. Barker, J. Ellis, E. Grassam, A. Cotterill, W. Fisher, W. Feather, A theoretical and experimental study of light absorption and scattering by in vivo skin. Phys Med Biol, 25(1980), pp. 695-709 
[17] J. Sun, M. L. Smith, L. N. Smith, A. Farooq, Compensation of illumination radiance in photometric stereo, IEEE International Conference on Machine Vision, Dec. 2010, Hong Kong, China

[18] N. Tsumura, Y. Miyake, F.H. Imai, Medical vision: measurement of skin absolute spectral-reflectance-image and the application to component analysis, Third Int. Conf. Multispectral Colour Sci., (2001), pp. 25-28

[19] M. Vilaseca, J. Pujol, M. Arjona, F.M. Martinez-Verdu, Color visualization system for near-infrared multispectral images, Journal of Imaging Science and Technology, (2005), pp. 246-255

[20] D. Lu, E. Moran, M. Batistella. Linear mixture model applied to Amazonian vegetation classification, Remote Sensing of Environment, (2003), pp. 456-469

[21] H. Takiwaki, et. al, Quantification of erythema and pigmentation using a videomicroscope and computer, Br. J. Dermatol., 131(1984), pp. 85-92

[22] B.D.Alessandro and A.P.Dhawan, 3D volume reconstruction of skin lesions for melanin and blood volume estimation and lesion severity analysis, IEEE Transactions on Medical Imaging, 31(11), (2012), pp.2083-2092

[23] V.P. Zharov, S. Ferguson, J.F. Eidt, P.C. Howard, L.M. Fink, M. Waner, Infrared imaging of subcutaneous veins, Lasers in Surgery and Medicine, 34(2004), pp. 56-61

[24] C. Fredembach, N. Barbuscia, S. Susstrunk, Combining visible and near-infrared images for realistic skin smoothing, IS\& T/SID 17th Color Imaging, 2009

[25] N. Tsumura, M.N Dang, Y. Miyake, Estimating the directions to light sources using images of eye for reconstructing 3D human face, IS\&T/SID's 11th Color Imaging, 2003 